\title{
Dynamic tracing of immune cells in an orthotopic gastric carcinoma mouse model using near-infrared fluorescence live imaging
}

\author{
XIAOHUI DU ${ }^{1,2}$, XIANGYU WANG ${ }^{3}$, NING NING ${ }^{1,2}$, SHAOYOU XIA $^{1,2}$, JUCHAO LIU $^{3}$, \\ WENTAO LIANG ${ }^{3}$, HUIWEI SUN ${ }^{3}$ and YINGXIN XU ${ }^{1,3}$ \\ ${ }^{1}$ General Surgery Department, Chinese PLA General Hospital, Beijing; ${ }^{2}$ General Surgery Department, \\ Chinese PLA General Hospital Hainan Branch, Hainan; ${ }^{3}$ Institute of General Surgery, \\ Chinese PLA General Hospital, Beijing, P.R. China
}

Received February 15, 2012; Accepted May 4, 2012

DOI: $10.3892 /$ etm.2012.579

\begin{abstract}
Adoptive cellular immunotherapy (ACI) has been demonstrated to be a promising cancer therapeutic, however, the distribution of immune cells injected into a tumor-bearing body is unclear. In this study, we investigated the tumor-targeting capacity of cytokine-induced killer (CIK) cells and cytotoxic T lymphocytes (CTLs) in a human gastric carcinoma orthotopic mouse model using a near-infrared fluorescence imaging system. CIK cells and tumor-specific CTLs were prepared with the near-infrared fluorescent dye DiR. As expected, no significant change in the proliferation rate or antitumor activity of CIK cells and CTLs was noted after labeling with DiR. Furthermore, a gastric carcinoma orthotopic model was established using a fibrinogen-thrombin method in nude mice followed by intraperitoneal infusion of the labeled immune cells into nude mice with established gastric carcinoma. Dynamic tracing of the immune cells was performed using a fluorescencebased live imaging system. Concentrated fluorescence signals were observed for a minimum of two weeks at the tumor site in mice infused with either CIK cells or CTLs with a peak signal at $48 \mathrm{~h}$. Notably, CTLs were more persistent at the tumor site and exhibited a more intense antitumor activity than CIK cells following infusion. These results provided visual evidence of the tumor-targeting capacity of immune cells in live animals.
\end{abstract}

\section{Introduction}

The high morbidity and mortality of gastric cancer makes it particularly concerning. Recent advances in tumor immunology

Correspondence to: Professor Yingxin $\mathrm{Xu}$, General Surgery Department and Institute of General Surgery, Chinese PLA General Hospital, 28 Fuxing Road, Haidian, Beijing 100853, P.R. China E-mail: xuyingxin301@yeah.net

Key words: gastric carcinoma orthotopic model, cytokine-induced killer cells, cytotoxic $\mathrm{T}$ lymphocytes, antitumor effect, near-infrared fluorescence live imaging have led to the development of novel immunotherapies for cancer. Adoptive cellular immunotherapy (ACI), a developing cancer therapeutic, can mobilize and strengthen the body's immune system to kill cancer cells in both a specific and nonspecific manner. Furthermore, adverse effects stemming from ACI are milder than both radiotherapy and chemotherapy. While clinical trials using cancer vaccines have yielded low objective response rates (1), recent approaches based on ACI have shown significantly higher efficacy. Dudley et al demonstrated partial and complete responses in $\sim 50 \%$ of patients with metastatic melanoma treated with adoptive transfer of ex vivo-expanded autologous tumor-infiltrating lymphocytes and a lymphodepleting host conditioning regime $(2,3)$. While this method was demonstrated to improve quality of life and prolong survival time, the distribution of immune cells injected into a tumorbearing body has yet to be described. Furthermore, the ability of intraperitoneally infused immune cells to target in situ gastric cancer is also unknown. Thus, it would be worthwhile to find visual evidence of the tumor-targeting ability of immune cells.

With modern fluorescence imaging techniques, we are able to trace the migration of cells in living animal models. Unfortunately, the results of monitoring immune cells injected into the gastric cancer orthotopic model were unsatisfactory due to a low signal-to-noise ratio (4). In recent years, however, the development of a near-infrared fluorescence imaging technique has made it possible to better trace living cells in deep tissue (5).

In the present study, we established an orthotopic gastric carcinoma nude mouse model and dynamically monitored cytokine-induced killer (CIK) cells and cytotoxic T lymphocytes (CTLs) labeled with the near-infrared fluorescent dye, DiR (1,1'-dioctadecyl-3,3,3',3'-tetramethyl indotricarbocyanine iodide).

\section{Materials and methods}

Cell line. Human gastric adenocarcinoma cell line BGC-823 (maintained in our laboratory) was grown in Dulbecco's modified Eagle's medium (DMEM) (Sigma, St. Louis, MO, USA) with $10 \%$ fetal bovine serum and $1 \mathrm{U} / \mathrm{ml}$ gentamicin and maintained in a humidified atmosphere of $5 \% \mathrm{CO}_{2}$ at $37^{\circ} \mathrm{C}$. 
CIK cells were generated from the peripheral blood mononuclear cells (PBMCs) of volunteers.

Animals. Female BALB/c-nu/nu nude mice (6- to 8-weeks old) were obtained from the animal center of the Academy of Military Medical Science (Beijing, China). All animals were housed under specific pathogen-free conditions and all animal protocols followed the experimental procedures of the National Institutes of Health Guide for the Care and Use of Laboratory Animals. Water and food were available ad libitum. Sterile supplies and techniques were applied whenever animals underwent surgery.

Preparation of immune cells. From volunteers who signed an informed consent, $10-20 \mathrm{ml}$ of peripheral blood was drawn into sterile centrifuge tubes containing heparin. PBMCs were obtained from buffy coats by Ficoll-Hypaque density centrifugation, washed with saline and then transferred to sterile Petri dishes with fresh serum-free Cellix 901 medium and incubated at $37^{\circ} \mathrm{C}$ in a humidified atmosphere of $5 \% \mathrm{CO}_{2}$.

CIK cells. Three hours later, cells in suspension were transferred to fresh dishes containing serum-free Cellix 601 medium with $1,000 \mathrm{U} / \mathrm{ml}$ recombinant human IFN- $\gamma$ at a concentration of $2 \times 10^{6}$ cells $/ \mathrm{ml}$. The next day, immobilized anti-CD3 antibody $(2 \mu \mathrm{g} / \mathrm{ml})$, anti-CD28 antibody $(1 \mu \mathrm{g} / \mathrm{ml})$ and recombinant human IL-2 $(1,000 \mathrm{U} / \mathrm{ml})$ were added to the incubation medium. On Day 5, fresh serum-free Cellix 602 medium containing recombinant human IL-2 $(1,000 \mathrm{U} / \mathrm{ml})$ was added to the cell suspension and replenished every 2-3 days over 9 more days of incubation. During the generation period, cell number was maintained at approximately $5 \times 10^{6} / \mathrm{ml}$.

CTLs. Following $3 \mathrm{~h}$ of PBMCs incubation in a sterile Petri dish, as previously described, the adherent cells were incubated in Cellix 901 medium containing recombinant human IL-4 $(1,000 \mathrm{IU} / \mathrm{ml})$ and GM-CSF $(1,000 \mathrm{IU} / \mathrm{ml})$ after being washed with Cellix 901 medium. On Day 7, BGC-823 cell lysate $(20 \mu \mathrm{g} / \mathrm{ml})$, which was obtained by repeated freezing-thawing, and Pseudomonas aeruginosa $(3 \mu \mathrm{l} / \mathrm{ml})$ were added to the incubation medium. The next day, the cells were obtained and co-cultured with the PBMC suspension cells following the same incubation conditions as that of the CIK cells.

Fluorescent labeling of immune cells. DiR (1,1'-dioctadecyl3,3,3',3'-tetramethyl indotricarbocyanine iodide) is a lipophilic, near-infrared fluorescent cyanine dye useful for labeling the cytoplasmic membrane. Our laboratory found that the proliferation rate and tumor-killing activity of immune cells were not significantly affected by the use of $10 \mu \mathrm{g}$ of DiR/ $10^{6}$ cells at a concentration of $1 \times 10^{6}$ cells $/ \mathrm{ml}$. Thus, this is a useful labeling technique for immune cell tracing experiments.

CIK cells and CTLs were put in suspension at a concentration of $1 \times 10^{6} \mathrm{cells} / \mathrm{ml}$. The DiR working solution $(1 \mu \mathrm{g} / \mu \mathrm{l})$ was added into the cell suspension and incubated for $30 \mathrm{~min}$ at $37^{\circ} \mathrm{C}$ with $10 \mu \mathrm{l}$ of dye $/ 10^{6}$ cells. The dye was then cleared away with two washes of Cellix 602 medium followed by centrifugation at 1,700 rpm for $8 \mathrm{~min}$. Flow cytometry (Beckman Coulter) was applied to verify staining using near-infrared excitation. A trypan blue exclusion test was run to determine viability, and the viable cells were brought to the desired concentration in sterile saline.

\section{Cell viability assay}

Proliferation assay. An MTT assay was used to detect the proliferation of immune cells. Single-cell suspensions of immune cells were made both before and after fluorescent labeling at a cell concentration of $5 \times 10^{5}$ cells $/ \mathrm{ml}$ in Cellix 602 medium containing recombinant human IL-2 $(1,000 \mathrm{U} / \mathrm{ml})$. Each suspension was added to a 96-well culture plate at $100 \mu \mathrm{l} /$ well with 4 groups/suspension and triplicate wells/group then cultured in a humidified atmosphere of 5\% $\mathrm{CO}_{2}$ at $37^{\circ} \mathrm{C}$. On Days $1,3,6$ and 10 one group from each suspension was tested respectively as follows: i) $30 \mu 1$ of MTT solution was added into each test well and incubated for $4 \mathrm{~h}$, ii) the suspension was centrifuged at 1,500 rpm for $5 \mathrm{~min}$ and the supernatant was discarded, iii) $100 \mu \mathrm{l}$ of DMSO was added to each well and agitated gently for $20 \mathrm{~min}$ and iv) the absorbance value of each well was measured by an automatic ELISA reader at a wavelength of $492 \mathrm{~nm}$.

Cytotoxicity assay. A lactate dehydrogenase (LDH) release assay was used to determine the cytotoxicity of the immune cells. The ratios of effect to target $(\mathrm{E} / \mathrm{T})$ cells were set at $5: 1$, 10:1, 20:1 and 40:1. Target cells (BGC-823 cells) and effect cells (CIK cells and CTLs, before and after labeling) were added to a 96-well culture plate with triplicate wells/suspension ratio and incubated for $24 \mathrm{~h}$ in a humidified atmosphere of $5 \% \mathrm{CO}_{2}$ at $37^{\circ} \mathrm{C}$. After centrifugation of the suspension at $1,700 \mathrm{rpm}$ for $4 \mathrm{~min}, 50 \mu \mathrm{l}$ was removed and mixed with $50 \mu \mathrm{l}$ of substrate solution and then incubated for $30 \mathrm{~min}$ in the dark. Finally, $50 \mu$ l of stop solution was added and the absorbance value of each well was measured using an automatic ELISA reader at a wavelength of $492 \mathrm{~nm}$.

Preparation of the gastric carcinoma orthotopic model. One female nude mouse was injected subcutaneously (s.c.) with $1 \times 10^{7}$ BGC-823 cells. When a tumor formed one week later, we anesthetized the mouse and surgically excised the tumor. The tumor tissue was further cut into fragments of $\sim 1 \mathrm{~cm}^{3}$. Another nude mouse was then anesthetized and a left paramedian abdominal incision was made by which we carefully drew out the stomach, which was almost covered by the liver, and made a few slight scarifications with a needle on the serosal surface near the greater curvature. One piece of tumor fragment was placed over the scarifications and a single $10 \mu \mathrm{l}$ drop of fibrinogen solution was applied to cover it followed by another single $10 \mu \mathrm{l}$ drop of thrombin solution $\sim 5 \mathrm{sec}$ later. When the gelatinous material formed, the stomach was carefully returned to its position in the body and the incision was closed. All steps were carried out aseptically.

\section{Adoptive transfer and fluorescence live imaging}

Experimental design. Nude mice bearing in situ gastric carcinomas (4-6 weeks after being implanted with tumor fragments) were randomized into groups: (i) intraperitoneal injection of CTLs (CTL-i.p.) and (ii) intraperitoneal injection of CIK cells (CIK-i.p.). Saline suspensions of CTLs and CIK cells labeled with DiR were constructed at a concentration of $1 \times 10^{8}$ cells $/ \mathrm{ml}$ and each tumor-bearing mouse received an infusion of $0.1 \mathrm{ml}$ $\left(1 \times 10^{7}\right)$ of labeled cells.

Fluorescence live imaging (FLI). After infusion of mice with CTLs or CIK cells, each mouse was anesthetized with isoflurane and FLI was performed using the Xenogen 

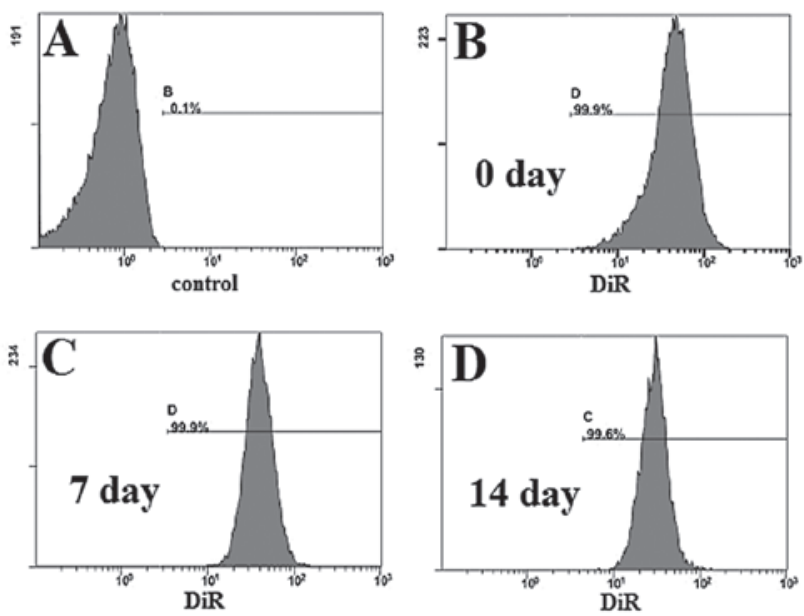

Figure 1. Percentage of fluorescently labeled immune cells. (A) Control group, (B) detection on the day of staining (Day 0), (C) detection on Day 7 and (D) dectecion on Day 14

IVIS-Spectrum Imaging System (Xenogen; Caliper Life Sciences, Inc.). Imaging examination times were set as follows: immediately after infusion (Day 0), 24 h (Day 1), 48 h (Day 2), $72 \mathrm{~h}$ (Day 3), 6 days, 10 days and 14 days after infusion.

Statistical analysis. Data were analyzed with a MannWhitney U test or ANOVA with Bonferroni post-test correction using GraphPad Prism v.5 software (GraphPad Software).

\section{Results}

Generation of near-infrared fluorescent-labeled immune cells with DiR. To determine the labeling efficiency of our DiR system, we used a five channel flow cytometer (Beckman Coulter) to detect and calculate the percentage of labeled immune cells (Fig. 1). Fluorescence was detected, and the labeling efficiency was as high as $99.9 \%$, when compared to the control group (unlabeled immune cells), in the cy7 channel (Fig. 1A and B). On Days 7 and 14, labeling efficiency remained as high as $99.9 \%$ (Fig. 1C) and 99.6\% (Fig. 1D), respectively.

In order to determine whether DiR influences the proliferation of immune cells, an MTT assay was used. CIK cells and CTLs were stained by DiR on Day 0, and on Days 1, 3, 6 and 10 we detected and compared the OD value of labeled and unlabeled immune cells. Proliferation curves were drawn according to these OD values (Fig. 2). Proliferation was not significantly altered after labeling with DiR.
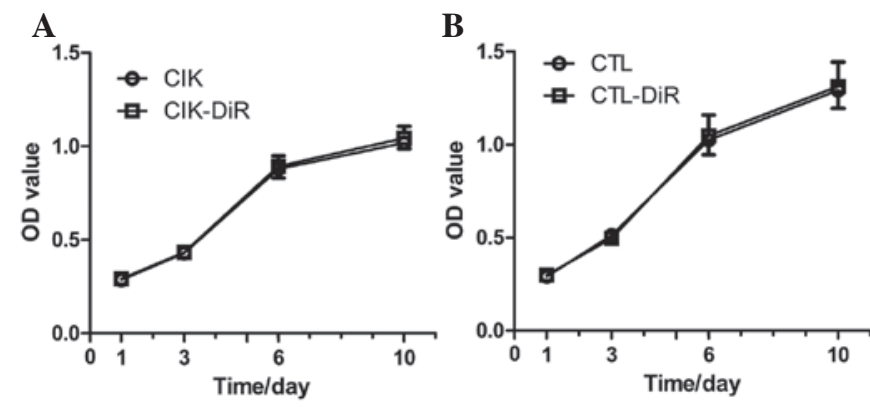

Figure 2. Proliferation curves of immune cells labeled and unlabeled with DiR (n=4). (A) CIK cells and (B) CTLs.

The antitumor effect of immune cells was determined by an $\mathrm{LDH}$ release assay. CIK cells and CTLs were tested at E/T ratios of 5:1, 10:1, 20:1 and 40:1. The tumor cell-killing rates were not significantly different between labeled and unlabeled CIK cells or between labeled and unlabeled CTLs at each E/T ratio $(\mathrm{P}>0.05)$ (Table I). However, CTLs exhibited a stronger antitumor activity than CIK cells at each $\mathrm{E} / \mathrm{T}$ ratio $(\mathrm{P}<0.05)$.

Tumor targeting of immune cells in vivo. DiR-labeled CTLs and CIK cells were injected intraperitoneally into tumorbearing nude mice. FLI was performed at different time points over the next two weeks (Fig. 3A). The signal was limited to the abdominal area during the initial period (Day 0). The strongest signal was detected at the injection site with a slight signal also observed in the intestines. We focused our attention on the tumor area. By the time we employed FLI $24 \mathrm{~h}$ after the initial intraperitoneal injection (Day 1), the strongest signal had migrated to the tumor area, although a slight signal was still detected in other parts of the mouse. The signal in the tumor area increased and remained strong through $72 \mathrm{~h}$ postinjection (Days 2 and 3). From Day 6 on, the signal in the tumor area began to gradually subside. The distributions of CIK cells and CTLs were similar, in general; however, CTLs exhibited a stronger tumor-targeting ability than CIK cells at each time point. In order to further compare the tumor-targeting ability of CIK cells and CTLs, several mice were sacrificed and their tumors were separated and imaged on Days 2 and 14 (Fig. 3B). Living Image v.4.1 software was used to draw and calculate the region of interest (ROI). We found that the signal from the tumor tissue in the CTL group was higher than that of the CIK group on Days 2 and 14 ( $\mathrm{P}<0.05)$ (Fig. 3C). We also imaged other organs, such as the liver, spleen, intestines and lung on Day 14 and confirmed the signals of these organs (Fig. 3D).

Table I. Antitumor effects of CIK cells and CTLs prior to and after labeling in vitro.

\section{$5: 1$}

$20.75 \pm 0.67$

$20.7 \pm 1.78$

CIK-DiR

CTL $25.32 \pm 2.40$

$26.89 \pm 0.56$
$10: 1$

20:1 40:1

$41.03 \pm 2.84$
$41.44 \pm 2.52$
$49.99 \pm 4.14$
$49.3 \pm 2.92$

$41.03 \pm 2.84$

$49.99 \pm 4.14$
$49.3 \pm 2.92$
$69.49 \pm 6.06$

$69.35 \pm 5.29$

$86.47 \pm 5.28$

$87.95 \pm 4.61$
$61.7 \pm 2.64$

$69.55 \pm 3.82$

$68.91 \pm 3.94$ 
A

a)

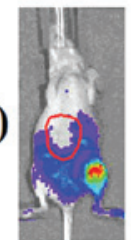

b)

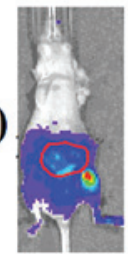

day 0
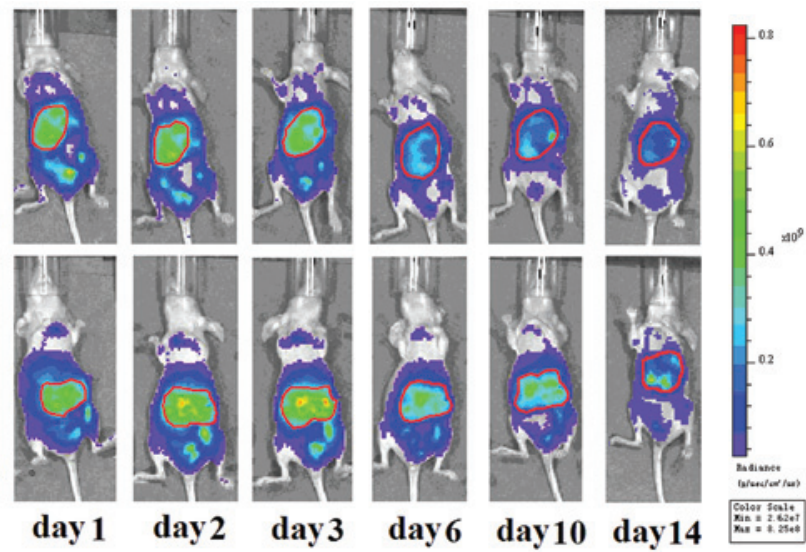

B
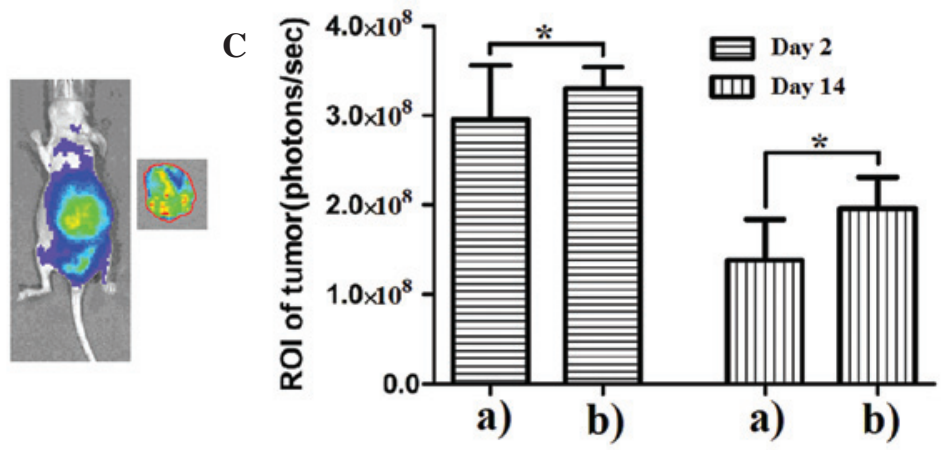

D tumor liver spleen intestine lung

a)

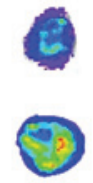

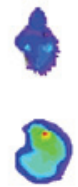
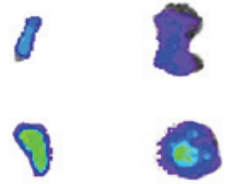

Figure 3. Fluorescence live imaging (FLI) of immune cells trafficking in vivo. (A) Distribution of immune cells in vivo, (B) separation of tumor tissue and fluorescence imaging in vitro, (C) comparison of tumor ROI between CIK cell and CTL infusion groups on Days 2 and 14 , (D) fluorescence imaging of organs on Day 14. a, CIK cell infusion group; b, CTL infusion group.

\section{Discussion}

This study demonstrates the tumor-targeting capacity of CIK cells and CTLs following intraperitoneal infusion. There is a need to visualize the migration of immune cells injected into living animals and we achieved this by using a new near-infrared fluorescent dye, DiR (1,1'-dioctadecyl-3,3,3',3'tetramethyl indotricarbocyanine iodide), although the use of $\mathrm{DiR}$ as an in vivo cell tracer is still in its initial experimental stages. Kalchenko et al (6) successfully used DiR to stain human leukemia G2L cells, mouse lymphocytes and rat red blood cells for in vivo tracer experiments. Granot et al (7) also used DiR to mark fibroblast cells, which were then observed targeting to an ovarian cancer tumor some distance away from the injection site. The properties of near-infrared wavelengths make them ideal for imaging in deep tissue. Our results showed that labeling with DiR had no significant influence on the biological properties of CIK cells and CTLs, suggesting DiR to be suitable for use in living animal experiments. This finding is consistent with recent studies employing a similar lipophilic carbocyanine dye DiI $(8,9)$.
Adoptive cellular immunotherapy (ACI), a modern treatment strategy for cancer, has lately received increased attention (10-14). CIK cells, a new generation of antitumor adoptive immune cells following the steps of lymphokine-activated killer cells (LAK cells), tumor-infiltrating lymphocytes (TILs) and anti-CD3 monoclonal antibody-activated killer cells (CD3AK cells), are one of the most widely used ACI cell type. With their characteristic rapid rate of proliferation and high efficiency and broad spectrum tumor cell-killing ability, CIK cells are generally regarded as a safe and efficient cell type for use in tumor immunotherapy (15). CIK cells have been shown to kill tumor cells in a non-MHC-restricted manner, similar to NK cells requiring no specific antigen recognition (16-18). The mechanism of CIK cells' antitumor activity has not yet been described; however, evidence shows that BLT (N-benzylcarbonyl-L-lysinethiobenzyl ester), perforin, cytolysin and other cytokines released by CIK cells play an important role (19). Our results indicate that CIK cells have a strong capacity to kill BGC-823 gastric cancer cells, with killing rates of $20.75 \pm 0.67,41.03 \pm 2.84,60.81 \pm 3.06$ and $69.49 \pm 6.06 \%$ at the effective target ratios of 5:1, 10:1, 20:1 and 
40:1, respectively. These results suggest that the number of immune cells migrating to the tumor site directly influence the antitumor effect.

The tumor-targeting capacity of tumor-specific $\mathrm{T}$ cells has been well described. Koya et al (20) utilized $\mathrm{T}$ cell receptor (TCR) engineering of mouse splenocytes to create specificity for tyrosinase, which is commonly expressed in melanoma cells. They genetically labeled the splenocytes with bioluminescence imaging (BLI) and positron emission tomography (PET) reporter genes to visualize the distribution and antigen-specific tumor-homing of these TCR transgenic $\mathrm{T}$ cells. Using a mouse tail vein infusion, they found that after an initial brief stage of systemic distribution, TCR-redirected $\mathrm{T}$ cells demonstrated an early pattern of specific distribution to antigen-matched tumors and locoregional lymph nodes followed by a more promiscuous distribution one week later with additional accumulation in antigen-mismatched tumors. Shu et al (21) used Micro-PET to detect and observe the movement of transplanted specific $\mathrm{CD}^{+} \mathrm{T}$ cells. They found that the tumor response could be predicted as early as three days following adoptive transfer via the tail vein and an increased signal was detected in mice exhibiting adoptive transfer cell proliferation. Adoptive transfer of CTLs has been performed in a number of clinical tests with impressive antitumor effects in patients with melanoma, breast cancer and renal carcinoma (22-25); however, research involving CTLs in gastric cancer is still lacking.

In our study, systemic distribution of CIK cells and CTLs injected intraperitoneally did not appear until $24 \mathrm{~h}$ post-injection, suggesting that CIK cells and CTLs can indeed infiltrate the circulatory system via the abdominal cavity, although the exact mechanism is not clear yet. After $24 \mathrm{~h}$, we observed systemic distribution of the immune cells with the strongest signal at the tumor site, indicating that CIK cells as well as CTLs can effectively migrate to the in situ gastric tumor with an excellent tumor-targeting capacity following intraperitoneal infusion. Furthermore, the signal at the tumor site gradually increased at 48 and $72 \mathrm{~h}$ post-injection, indicating that human immune cells could propagate after adoptive transfer into nude mice. Although CIK cells and tumor-specific CTLs were equally adept at targeting the tumor, ROI calculations of tumor tissue showed that the number of CTLs was significantly higher than the number of CIK cells at the tumor site $48 \mathrm{~h}$ and 14 days following adoptive transfer. We observed a similar distinction in other organs, such as the liver, spleen, intestines and lungs. These results suggest that tumor-specific CTLs are still the optimal immune cells for adoptive immunotherapy.

In conclusion, we provided visual evidence of the tumortargeting capacity of immune cells in live animals. This defined distribution pattern of adoptively transferred cells eliciting robust antitumor activity can be used to further analyze individual components of this combinatorial approach prior to the initiation of clinical trials.

\section{References}

1. Rosenberg SA, Yang JC and Restifo NP: Cancer immunotherapy: moving beyond current vaccines. Nat Med 10: 909-915, 2004.

2. Dudley ME, Wunderlich JR, Robbins PF, et al: Cancer regression and autoimmunity in patients after clonal repopulation with antitumor lymphocytes. Science 298: 850-854, 2002.
3. Dudley ME, Wunderlich JR, Yang JC, et al: Adoptive cell transfer therapy following non-myeloablative but lymphodepleting chemotherapy for the treatment of patients with refractory metastatic melanoma. J Clin Oncol 23: 2346-2357, 2005.

4. Frangioni JV: In vivo near-infrared fluorescence imaging. Curr Opin Chem Biol 7: 626-634, 2003.

5. He X, Gao J, Gambhir SS and Cheng Z: Near-infrared fluorescent nanoprobes for cancer molecular imaging: status and challenges. Trends Mol Med 16: 574-583, 2010.

6. Kalchenko V, Shivtiel S, Malina V, et al: Use of lipophilic nearinfrared dye in whole-body optical imaging of hematopoietic cell homing. J Biomed Opt 11: 050507, 2006.

7. Granot D, Addadi Y, Kalchenko V, Harmelin A, KunzSchughart LA and Neeman M: In vivo imaging of the systemic recruitment of fibroblasts to the angiogenic rim of ovarian carcinoma tumors. Cancer Res 67: 9180-9189, 2007.

8. Hemmrich K, Meersch M, von Heimburg D and Pallua N: Applicability of the dyes CFSE, CM-DiI and PKH26 for tracking of human preadipocytes to evaluate adipose tissue engineering. Cells Tissues Organs 184: 117-127, 2006.

9. Kikkawa YS and Pawlowski KS: Cochlear neuronal tracing for frequency mapping with DiI, NeuroVue, and Golgi methods. Acta Otolaryngol Suppl 559: 19-23, 2007.

10. Jiang J, Xu N, Wu C, et al: Treatment of advanced gastric cancer by chemotherapy combined with autologous cytokine-induced killer cells. Anticancer Res 26: 2237-2242, 2006.

11. Kim HM, Kang JS, Lim J, et al: Antitumor activity of cytokineinduced killer cells in nude mouse xenograft model. Arch Pharm Res 32: 781-787, 2009.

12. Toh U, Fujii T, Mishima M, et al: Conventional chemotherapy combined with the repetitive immune cell transfer for patients with refractory advanced gastric cancer. Gan To Kagaku Ryoho 34: 1931-1933, 2007 (In Japanese).

13. Wongkajornsilp A, Sangsuriyong S, Hongeng S, Waikakul S, Asavamongkolkul A and Huabprasert S: Effective osteosarcoma cytolysis using cytokine-induced killer cells pre-inoculated with tumor RNA-pulsed dendritic cells. J Orthop Res 23: 1460-1466, 2005.

14. Yamagishi H,Ueda Y and Oka T: A case report of immunotherapy on a patient with advanced gastric cancer by adoptive transfer of OK-432-reactive HLA-matched allogeneic lymphocytes. Cancer Immunol Immunother 46: 113-119, 1998.

15. Schmidt-Wolf IG, Lefterova P, Johnston V, Huhn D, Blume KG and Negrin RS: Propagation of large numbers of T cells with natural killer cell markers. Br J Haematol 87: 453-458, 1994.

16. Linn YC and Hui KM: Cytokine-induced NK-like T cells: from bench to bedside. J Biomed Biotechnol 2010: 435745, 2010.

17. Linn YC, Lau LC and Hui KM: Generation of cytokine-induced killer cells from leukaemic samples with in vitro cytotoxicity against autologous and allogeneic leukaemic blasts. Br J Haematol 116: 78-86, 2002.

18. Lopez RD, Waller EK, Lu PH and Negrin RS: CD58/LFA-3 and IL-12 provided by activated monocytes are critical in the in vitro expansion of $\mathrm{CD}_{56}{ }^{+} \mathrm{T}$ cells. Cancer Immunol Immunother 49: 629-640, 2001.

19. Mehta BA, Schmidt-Wolf IG, Weissman IL and Negrin RS: Two pathways of exocytosis of cytoplasmic granule contents and target cell killing by cytokine-induced $\mathrm{CD}^{+}{ }^{+} \mathrm{CD} 56^{+}$killer cells. Blood 86: 3493-3499, 1995.

20. Koya RC, Mok S, Comin-Anduix B, et al: Kinetic phases of distribution and tumor targeting by $\mathrm{T}$ cell receptor engineered lymphocytes inducing robust antitumor responses. Proc Natl Acad Sci USA 107: 14286-14291, 2010.

21. Shu CJ, Radu CG, Shelly SM, et al: Quantitative PET reporter gene imaging of $\mathrm{CD} 8^{+} \mathrm{T}$ cells specific for a melanoma-expressed self-antigen. Int Immunol 21: 155-165, 2009.

22. Butler MO, Lee JS, Ansen S, et al: Long-lived antitumor CD8 ${ }^{+}$ lymphocytes for adoptive therapy generated using an artificial antigen-presenting cell. Clin Cancer Res 13: 1857-1867, 2007.

23. Mackensen A, Meidenbauer N, Vogl S, Laumer M, Berger J and Andreesen R: Phase I study of adoptive T-cell therapy using antigen-specific $\mathrm{CD} 8^{+} \mathrm{T}$ cells for the treatment of patients with metastatic melanoma. J Clin Oncol 24: 5060-5069, 2006.

24. Yamaguchi Y, Ohshita A, Hironaka K, et al: Adoptive immunotherapy using autologous lymphocytes sensitized with HLA class I-matched allogeneic tumor cells. Oncol Rep 16: 165-169, 2006.

25. Yee C, Thompson JA, Byrd D, et al: Adoptive T cell therapy using antigen-specific $\mathrm{CD} 8^{+} \mathrm{T}$ cell clones for the treatment of patients with metastatic melanoma: in vivo persistence, migration, and antitumor effect of transferred T cells. Proc Natl Acad Sci USA 99: 16168-16173, 2002. 